\title{
Generalización de la ecuación de transformación de fases al análisis de la cinética de cristalización no-isoterma. Aplicación a la aleación vítrea $\mathrm{Cu}_{0.10} \mathrm{As}_{0.40} \mathrm{Se}_{0.50}$.
}

\author{
J. VÁZQUEZ, P.L. LÓPEZ-ALEMANY, P. VILLARES Y R. JIMÉNEZ-GARAY. \\ Departamento de Física de la Materia Condensada. Facultad de Ciencias. Universidad de Cádiz. 11510. Puerto Real (Cádiz).
}

\begin{abstract}
Las técnicas de análisis térmico diferencial se emplean con frecuencia para estudiar la cinética de las reacciones de transformación y, en particular la de la cristalización de aleaciones vítreas. Los datos térmicos correspondientes pueden analizarse por el método de Kissinger, que se desarrolló originalmente para el estudio de reacciones homogéneas. Durante varias décadas, el consenso en la literatura fue que tales aplicaciones (es decir, a transformaciones heterogéneas de estado sólido) del método de Kissinger no eran válidas. En el presente trabajo se señalan las principales objeciones a las aplicaciones mencionadas y se da una deducción alternativa de los resultados teóricos. Estos resultados demuestran que el método de Kissinger es válido para reacciones heterogéneas del tipo descrito por la ecuación de Johnson-Mehl-Avrami en el caso isotermo. También se dan los datos de cristalización isoterma y no isoterma de la aleación vítrea $\mathrm{Cu}_{0.10} \mathrm{As}_{0.40} \mathrm{Se}_{0.50}$. Los resultados experimentales y la discusión realizada aquí, ayudan a aclarar los efectos del tiempo de incubación en la cinética de transformaciones no isotermas y proporcionan una demostración adicional de la validez de la teoría generalizada de Johnson-Mehl-Avrami para la descripción de transformaciones heterogéneas de estado sólido.
\end{abstract}

Palabras clave: Aleación vitrea, procesos isotermos y no isotermos, velocidad de calentamiento, parámetros cinéticos, recocido.

Generalization of the phase transformation equation for the analysis of non-isothermal crystallization kinetics. Application to the $\mathrm{Cu}_{0.10} \mathrm{As}_{0.40} \mathrm{Se}_{0.50}$ glassy alloy.

The techniques of differential thermal analysis are frequently employed to study the kinetics of the transformation reactions and, in particular, the crystallization of the glassy alloys. The corresponding thermal data can be analyzed by the Kissinger method, which was originally derived for the study of homogeneous reactions. The consensus in the literature, in several decades, was that such applications (i.e. to heterogeneous solid state transformations) of the Kissinger method are not valid. In the present work the principal objections to the mentioned applications are addressed and alternative derivations of theoretical results are provided. These results demonstrate that the Kissinger method is valid for heterogeneous reactions of the type described by Johnson-Mehl-Avrami equation in the isothermal case. Also the isothermal and non-isothermal data on crystallization of the $\mathrm{Cu}_{0.10} \mathrm{As}_{0.40} \mathrm{Se}_{0.50}$ glassy alloy are given. The experimental results and the discussion carried out here, help to clarify the effects of incubation time in non-isothermal transformation kinetics and provide a further demonstration of validity of the generalized Johnson-Mehl-Avrami theory for the description of heterogeneous solid state transformations.

Keywords: Glassy alloy, isothermal and non-isothermal processes, heating rate, kinetic parameters, preannealing.

\section{INTRODUCCIÓN}

El estudio de la cinética de cristalización en materiales vítreos ha estado limitado frecuentemente por la naturaleza elaborada de los procedimientos experimentales empleados. Sin embargo, el uso creciente de técnicas termoanalíticas, tales como el análisis térmico diferencial (ATD), o la calorimetría diferencial de barrido (DSC), ofrece la esperanza de obtener datos útiles con métodos sencillos. Es bien conocido el método termoanalítico desarrollado por Kissinger (1), que determina los parámetros cinéticos a partir de la representación del logaritmo del cociente $T_{\mathrm{p}}{ }^{2} / \beta$ ( $T_{\mathrm{p}}$ es la temperatura correspondiente a la máxima velocidad de cristalización y $\beta$ la velocidad de calentamiento) frente al recíproco de $T_{p}$ en experimentos no isotermos. Este método se ha usado con frecuencia para estu- diar la cristalización de aleaciones vítreas (2-8), a pesar de que la literatura sobre técnicas de análisis térmico ha manifestado el consenso de que la aplicación del método de Kissinger a reacciones de estado sólido no es adecuada. Sin embargo, un trabajo destacado de Henderson (9) ha proporcionado una base teórica para el tratamiento de técnicas de análisis no isotermas y justifica el empleo del método de Kissinger en muchas transformaciones de estado sólido. Las tres objeciones principales al uso de este método para estudiar reacciones de estado sólido son: 1. Los gradientes térmicos son inherentes a los métodos no isotermos. Por tanto es lógico pensar que resulten inexactitudes significativas por la aplicación del método de Kissinger, que no prevé la presencia de gradientes de tempera- 
tura. 2. La ecuación de la velocidad de reacción, que es apropiada para experimentos isotermos, se emplea en el análisis de Kissinger. Se argumenta con frecuencia que en el análisis de experimentos no isotermos debe incluirse un término, que considere la derivada parcial de la fracción transformada respecto a la temperatura; este punto se ha debatido en la literatura durante varios años. 3. El orden de la ecuación de reacción, considerado en el análisis de Kissinger es apropiado para transformaciones homogéneas (es decir, reacciones químicas en un gas) pero no es válido para las transformaciones heterogéneas, que generalmente ocurren en reacciones de estado sólido.

Respecto a la primera objeción, se pueden describir procedimientos sencillos para reducir la influencia de los gradientes de temperatura a niveles despreciables.

La confusión, que existe en la literatura acerca de la propia forma de la ecuación de la velocidad de reacción en el análisis de Kissinger (objeción 2), surge de considerar que la evolución de una reacción puede describirse como una función ordinaria de la temperatura y del tiempo. La fracción transformada es claramente una funcional (10), que depende de la historia térmica, y no una función ordinaria. Sin embargo, el análisis realizado en este trabajo, de acuerdo con una de las hipótesis del artículo de Henderson (9), pone de manifiesto que la velocidad de reacción es una función ordinaria de la temperatura y de la fracción transformada.

En cuanto a la tercera objeción, Henderson (9) ha demostrado que el método de Kissinger puede aplicarse al análisis de muchas reacciones heterogéneas. En el presente trabajo se describe un tratamiento alternativo de la cinética de transformaciones no isotermas. Dicho tratamiento pone de manifiesto que el método de Kissinger se puede aplicar a cualquier transformación del tipo descrito por la ecuación de Johnson-MehlAvrami (JMA) (11-14) en el caso isotermo. El tratamiento, que se presenta aquí, generaliza la ecuación JMA para tratar reacciones heterogéneas no isotermas, y demuestra que en el caso de velocidad de calentamiento constante se obtiene la relación de Kissinger, sin cometer errores apreciables. Finalmente, se analizan los datos de las cristalizaciones isoterma y no isoterma del semiconductor vítreo $\mathrm{Cu}_{0.10} \mathrm{As}_{0.40} \mathrm{Se}_{0.50}$, incluyéndose la influencia del tiempo de incubación sobre los experimentos no isotermos. Los datos concuerdan satisfactoriamente con los resultados teóricos.

\section{FUNDAMENTO TEÓRICO}

\subsection{Tratamiento isotermo}

La ecuación JMA (11-14) describe una amplia variedad de transformaciones de estado sólido y tiene la forma

$$
x(t)=1-\exp \left[-(K t)^{n}\right]
$$

donde $x$ es la fracción transformada en el tiempo $t, n$ es el exponente cinético y $K=K_{0} \exp (-E / R T)$ es la constante de velocidad de reacción con $K_{0}$ el factor de frecuencia, $E$ la energía de activación de la transformación , $R$ la constante de los gases y $T$ la temperatura absoluta.

La velocidad de reacción, $\mathrm{d} x / \mathrm{d} t$, se obtiene derivando la ec.[1] con respecto al tiempo, a temperatura constante, resultando

$$
\mathrm{d} x / \mathrm{d} t=n K^{n} t^{n-1}(1-x)=n K(1-x)[-\ln (1-x)]^{(n-1) / n}
$$

que es una función ordinaria de la temperatura y de la fracción transformada. Aunque estas velocidades pueden medirse a partir de los termogramas, los resultados se analizan más fácilmente tomando dos veces el logaritmo de la ec.[1] resultando la expresión

$$
\ln [-\ln (1-x)]=n \ln K+n \ln t \text {. }
$$

Para una temperatura dada, se pueden obtener los valores de $n$ y $K$ a partir de la pendiente y la ordenada en el origen, respectivamente, de la recta de regresión $\ln [-\ln (1-x)]$ frente a $\ln t$. Los valores de $\ln K$ se evalúan a diferentes temperaturas, repitiendo el procedimiento, y se obtienen los parámetros cinéticos $E$ y $K_{0}$ a partir de la representación lineal $\ln K$ frente a $1 / T$.

\subsection{Tratamiento no isotermo}

Resulta interesante la generalización de la ec.[1] para tratar experimentos en los que la temperatura es función del tiempo. Considerando que el mecanismo de reacción no cambia con la temperatura, puede admitirse razonablemente que $K t$ en la ec.[1] es proporcional al número de saltos atómicos entre estados energéticos diferentes en el intervalo de tiempo $t$ a temperatura $T$. Admitiendo que la evolución de la transformación la determina el número de saltos atómicos en el caso general (no isotermo), la ec.[1] se generaliza, dando

$$
x(t)=1-\exp \left[-\left(\int_{0}^{t} K\left[T\left(t^{\prime}\right)\right] \mathrm{d} t^{\prime}\right)^{n}\right]=1-\exp \left(-I^{n}\right)
$$

donde $K\left[T\left(t^{\prime}\right)\right]$ es función del tiempo a través de su dependencia de la temperatura tipo Arrhenius. Debe señalarse que la fracción transformada depende de $t \mathrm{y}$ de la historia térmica $T\left(t^{\prime}\right)$ para tiempos $t^{\prime}$ anteriores a $t$.

La velocidad de cristalización se obtiene derivando la ec.[4] con respecto al tiempo, resultando

$$
\mathrm{d} x / \mathrm{d} t=n K(1-x) I^{n-1}
$$

La máxima velocidad de cristalización se encuentra haciendo $\mathrm{d}^{2} x / \mathrm{d} t^{2}=0$, obteniéndose la relación

$$
\left.n K_{\mathrm{p}}\left(I^{n}\right)\right|_{\mathrm{p}}=\beta E I_{\mathrm{p}} / R T_{\mathrm{p}}^{2}+(n-1) K_{\mathrm{p}}
$$

en la que $\beta=\mathrm{d} T / \mathrm{d} t$ es la velocidad de calentamiento para un proceso no isotermo, y donde se denotan con el subíndice $p$ los valores de magnitudes correspondientes a la máxima velocidad de cristalización.

Usando la substitución $y^{\prime}=E / R T^{\prime}$ la integral $I$ puede evaluarse mediante la serie (15),

$$
-e^{-y^{\prime}} y^{\prime-2} \sum_{k=0}^{\infty} \frac{(-1)^{k}(k+1) !}{y^{\prime k}}
$$

donde se pueden tomar sólo los dos primeros términos, sin cometer error apreciable, ya que su cota es $c_{e}<3 ! \cdot y^{-2}$, porque en las series alternadas ésta es menor que el primer término despreciado. Si, además, se tiene en cuenta que en la mayoría de reacciones de cristalización $y=E / R T \geq 25$, el error es inferior al $1 \%$. Con la substitución y aproximación señaladas se obtiene para la máxima velocidad de cristalización 


$$
I_{\mathrm{p}}=R T_{\mathrm{p}}^{2} K_{\mathrm{p}}(\beta E)^{-1}\left(1-2 R T_{\mathrm{p}} / E\right)
$$

si se admite que $T_{0}<<T$ ( $T_{0}$ es la temperatura de comienzo del tratamiento térmico) para que pueda considerarse $y_{0} \rightarrow \infty$. Substituyendo la última expresión de $I_{\mathrm{p}}$ en la ec.[6], se obtiene una relación que, cuando se iguala a la ec.[7], da una ecuación cuya forma logarítmica es

$$
\ln \left(T_{p}^{2} / \beta\right)+\ln K_{0} R / E-E / R T_{p} \cong\left(2 R T_{p} / E\right)\left(1-1 / n^{2}\right)^{[8]}
$$

donde la función $\ln (1-z)$ con $z=2 R T_{\mathrm{p}} / n E$ ó $z=2 R T_{\mathrm{p}} / E$ se ha desarrollado en serie y sólo se ha tomado el primer término de la misma.

Hay que señalar que la ec.[8] se reduce a la expresión de Kissinger para $n=1$. Además, el segundo miembro de la ec.[8] es despreciable en comparación con los términos del primero en la mayoría de reacciones de cristalización a velocidades de calentamiento normales $\left(\leq 100 \mathrm{Kmin}^{-1}\right)$. Esto pone de manifiesto que el método de Kissinger es apropiado para analizar no sólo reacciones homogéneas sino también heterogéneas descritas por la ecuación (JMA) en experimentos isotermos. En estas condiciones la ec.[8] con el segundo miembro nulo, puede usarse para calcular $E$ y $K_{0}$ a partir de la pendiente y la ordenada en el origen de una representación $\ln \left(T_{p}{ }^{2} / \beta\right)$ frente $1 / T_{p}$.

Finalmente, considerando $E / R T_{p}>1$ (usualmente $E /$ $R T_{\mathrm{p}} \geq 25$ ) (16) en la ec.[7] y relacionándola con la ec.[6], resulta $I_{\mathrm{p}}=1$, que permite obtener, a partir de la ec.[5] la expresión

$$
n=(\mathrm{d} x / \mathrm{d} t)_{\mathrm{p}} R T_{p}^{2}(0.37 \beta E)^{-1}
$$

con la que se puede calcular el exponente cinético.

\section{PROCEDIMIENTO EXPERIMENTAL}

La aleación semiconductora $\mathrm{Cu}_{0.10} \mathrm{As}_{0.40} \mathrm{Se}_{0.50}$ se obtuvo en forma másica a partir de sus elementos del $99.999 \%$ de pureza, que se introdujeron en una ampolla de cuarzo sellada a una presión de $10^{-2} \mathrm{~N} \mathrm{~m}^{-2}$. Esta ampolla se mantuvo en un horno rotatorio a $1223 \mathrm{~K}$ durante $3 \mathrm{~h}$. y luego se enfrió bruscamente al aire para evitar la cristalización del material. La naturaleza vítrea del mismo se comprobó con un barrido de rayos- $X$ en un difractómetro Siemens D500. El comportamiento térmico se investigó usando un calorímetro diferencial de barrido PerkinElmer DSC7 con precisión de $\pm 0.1 \mathrm{~K}$. Las muestras pulverizadas, con masa aproximada de $20 \mathrm{mg}$ y tamaño de partícula alrededor de $40 \mu \mathrm{m}$, se introdujeron en cápsulas de aluminio, utilizando otra de estas cápsulas vacía como referencia. Se mantuvo un flujo constante $\left({ }^{\mathrm{a}} 60 \mathrm{ml} \cdot \mathrm{min}^{-1}\right)$ de nitrógeno a través del horno del calorímetro con objeto de extraer los gases emitidos por la reacción, que son muy corrosivos para el equipo sensor instalado en dicho horno. Los datos cinéticos de las transformaciones isotermas se obtuvieron registrando la salida, $Q_{\mathrm{DSC}}$ del calorímetro como función del tiempo para una serie de temperaturas. Considerando que $Q_{\mathrm{DSC}}$ es proporcional a la velocidad de reacción, la fracción cristalizada se puede expresar como

$$
x(t)=\frac{1}{A} \int_{0}^{t} Q_{\mathrm{DSC}} d t^{\prime}=\frac{A(t)}{A}
$$

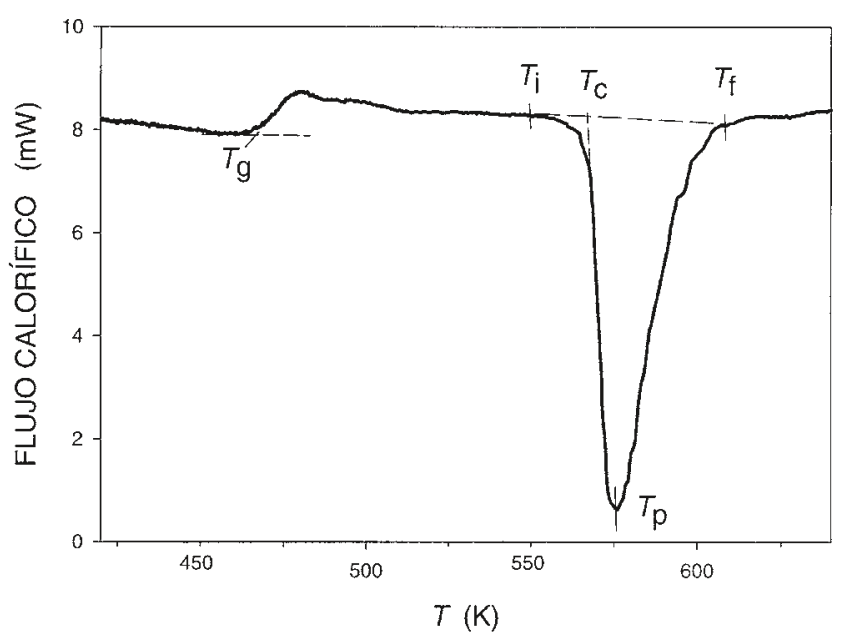

Fig.1. Gráfica DSC típica de la aleación $\mathrm{Cu}_{0.10} \mathrm{As}_{0.40} \mathrm{Se}_{0.50}$ para una velocidad de calentamiento de $16 \mathrm{~K} \mathrm{~min}^{-1}$.

donde $A=A(\infty)$. Por tanto, la técnica para obtener los parámetros $n$ y $K(T)$ en experimentos isotermos consiste en representar $\ln \{\ln [A /(A-A(t))]\}$ frente a $\ln t$ siendo $n$ la pendiente $\mathrm{y}$ $K(T)=t_{0}{ }^{-1}$ con $t_{0}$ el tiempo para que $\ln \{\ln [A /(A-A(t))]\}=0$. Los datos cinéticos de las transformaciones no isotermas se tomaron a partir de barridos de las muestras desde la temperatura ambiente a velocidades de calentamiento de 2, 4, 8, 16, 32 y 64 $\mathrm{K} \mathrm{min}{ }^{-1}$. El termograma de la aleación estudiada a $\beta=16 \mathrm{~K} \mathrm{~min}^{-1}$ representado en la Fig.1, muestra la temperatura de transición vítrea, $T_{\mathrm{g}}=465.8 \mathrm{~K}$, la temperatura de inicio de la cristalización, $T_{\mathrm{c}}=568.0 \mathrm{~K}$, y la correspondiente al pico de cristalización, $T_{\mathrm{p}}$ $=575.3 \mathrm{~K}$. Este comportamiento es típico de una transformación vidrio-cristal. Se observa que los valores de $T_{\mathrm{g}^{\prime}} T_{\mathrm{c}} \mathrm{y} T_{\mathrm{p}}$ crecen con las $\beta$ crecientes como sucede frecuentemente en la literatura (17).

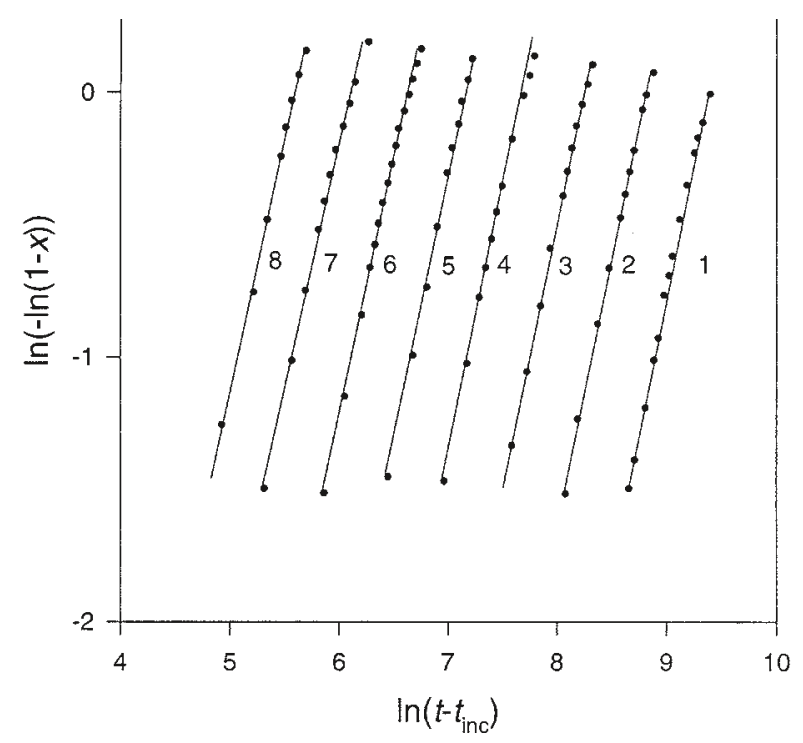

Fig.2. Gráficas para obtener el exponente cinético, $n$, de la ec.[1], como se describe en el texto. Los parámetros característicos, correspondientes a cada recta, se dan en la Tabla I. 
TABLA I

PARÁMETROS TEÓRICOS Y EXPERIMENTALES OBTENIDOS POR TRATAMIENTOS ISOTERMO Y DE CALENTAMIENTO CONTINUO DE LA ALEACIÓN VítREACu ${ }_{0.10} \mathrm{As}_{0.40} \mathrm{Se}_{0.50}$

\begin{tabular}{|c|c|c|c|c|c|c|c|c|c|c|c|}
\hline \multicolumn{4}{|c|}{ Isotermo } & \multicolumn{8}{|c|}{ Calentamiento continuo } \\
\hline Barrido & $T(\mathrm{~K})$ & $t_{\text {inc }}(\mathrm{s})$ & $n$ & Barrido & $\beta(\mathrm{K} / \mathrm{min})$ & $\begin{array}{c}T_{\mathrm{p}}(\mathrm{K}) \\
{[\mathrm{Exp} .(\mathrm{a})]}\end{array}$ & $\begin{array}{c}T_{\mathrm{p}}(\mathrm{K}) \\
{[\operatorname{Exp} .(\mathrm{b})]}\end{array}$ & $\begin{array}{r}T_{\mathrm{p}}(\mathrm{K}) \\
\text { (Teórica) }\end{array}$ & $\begin{array}{l}\operatorname{ATMM}(\mathrm{K}) \\
{[\operatorname{Exp} \cdot(\mathrm{a})]}\end{array}$ & $\begin{array}{c}\operatorname{ATMM}(\mathrm{K}) \\
{[\operatorname{Exp} .(\mathrm{b})]}\end{array}$ & $\begin{array}{c}\text { ATMM (K) } \\
\text { (Teórica) }\end{array}$ \\
\hline 1 & 513 & 2573 & 2.06 & 1 & 2 & 554.2 & 548.2 & 547.0 & 7.2 & 10.8 & 11.9 \\
\hline 2 & 518 & 1240 & 2.03 & 2 & 4 & 561.0 & 554.9 & 553.7 & 8.1 & 11.5 & 12.2 \\
\hline 3 & 523 & 459 & 2.01 & 3 & 8 & 568.0 & 561.6 & 560.5 & 8.1 & 11.5 & 12.5 \\
\hline 4 & 528 & 201 & 1.95 & 4 & 16 & 575.3 & 568.4 & 567.5 & 8.2 & 12.5 & 12.8 \\
\hline 5 & 533 & 111 & 1.98 & 5 & 32 & 582.6 & 575.4 & 574.6 & 9.0 & 12.5 & 13.2 \\
\hline 6 & 538 & 55 & 1.93 & 6 & 64 & 590.2 & 582.6 & 582.0 & 11.1 & 13.3 & 13.4 \\
\hline 7 & 543 & 39 & 1.84 & & & & & & & & \\
\hline 8 & 548 & 20 & 1.90 & & & & & & & & \\
\hline
\end{tabular}

\section{RESULTADOS Y DISCUSIÓN}

Las medidas calorimétricas muestran un tiempo de incubación, $t_{\text {inc }}$, durante el que no ocurren transformaciones observables. La dependencia térmica de $t_{\text {inc }}$ puede describirse como un proceso activado con una energía próxima a la de la transformación de fase; lo que se observa con frecuencia en estudios de cristalización (18).

Los termogramas isotermos correspondientes a un conjunto de temperaturas se integraron para obtener las gráficas de $\ln [-$ $\ln (1-x)]$ frente a $\ln \left(t-t_{\text {inc }}\right)$ mostradas en la Fig.2, que presentan una buena linealidad. Los valores del parámetro $n$ determinados a partir de la Fig. 2 y dados en la Tabla I para diferentes temperaturas, oscilan entre 1.84 y 2.06 con un valor medio de 1.96. El logaritmo de la constante de velocidad, $K$, obtenido de la Fig. 2 se ha representado frente a $1 / T$ en la Fig.3. La pendiente de la línea, obtenida mediante ajuste por mínimos cuadrados, da $E=60.9 \mathrm{kcal} \mathrm{mol}^{-1}$ para la energía de activación y la ordenada en el origen de la mencionada línea da $K_{0}=5.11 \times 10^{21}$ $\mathrm{s}^{-1}$ para el factor de frecuencia.

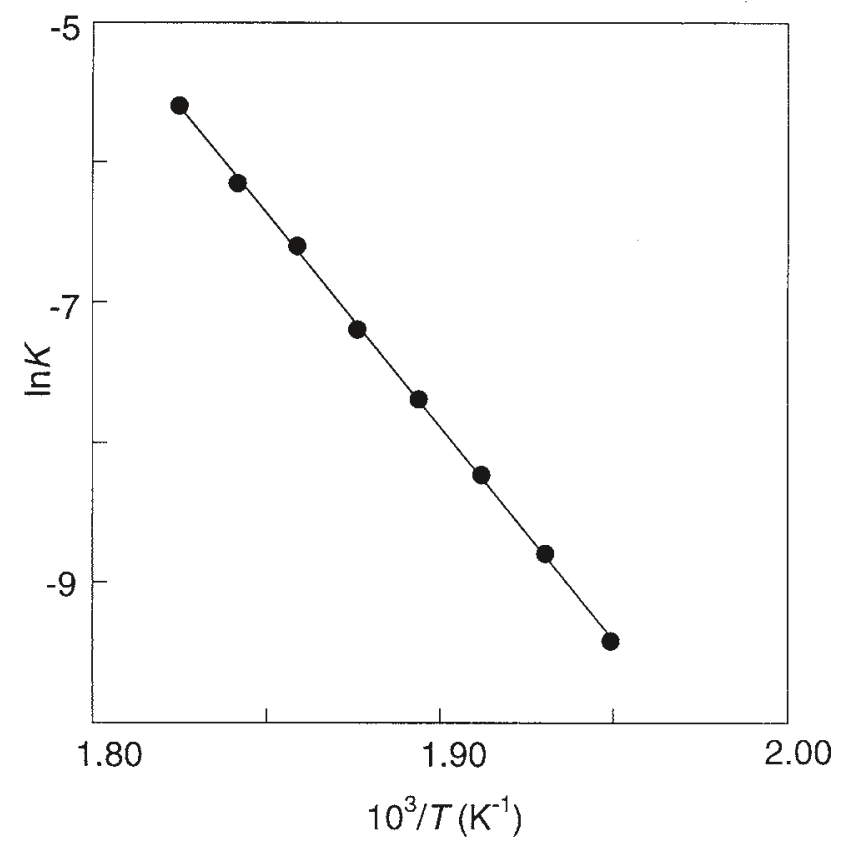

Fig.3. El logaritmo de la constante de velocidad de reacción, $K$, obtenido a partir de medidas isotermas, se representa frente a $1 / T$.
Los datos no isotermos de la aleación vítrea $\mathrm{Cu}_{0.10} \mathrm{As}_{0.40} \mathrm{Se}_{0.50^{\prime}}$ obtenidos a las velocidades de calentamiento dadas en la Tabla I, se analizaron usando la ec.[8] con el segundo miembro nulo y la ec.[9], deducidas en la subsección 2.2. La gráfica de $\ln \left(T_{\mathrm{p}}^{2} / \beta\right)$ frente a $1 / T_{p^{\prime}}$ mostrada en la Fig. 4 , se ajusta bien por una recta de la que se ha obtenido $E=60.6 \mathrm{kcal} \quad \mathrm{mol}^{-1} \mathrm{y} K_{0}=$ $1.90 \times 10^{21} \mathrm{~s}^{-1}$. El valor medio del exponente cinético es, de acuerdo con la ec.[9], $<n>=1.99$.

También se muestran en la Fig.4 los datos de calentamiento continuo correspondientes a un conjunto de muestras de la aleación $\mathrm{Cu}_{0.10} \mathrm{As}_{0.40} \mathrm{Se}_{0.50}$ recocidas previamente durante el tiempo de incubación (1200s a $518 \mathrm{~K}$ ), que se ha determinado en los experimentos isotermos. Se puede admitir que el recocido elimina el efecto de incubación en experimentos de calentamiento continuo permitiendo comparaciones directas con la teoría. Los valores resultantes para $E$ y $K_{0}$ son $62.5 \mathrm{kcal} \mathrm{mol}^{-1} \mathrm{y}$ $1.94 \times 10^{22} \mathrm{~s}^{-1}$, respectivamente, que están de acuerdo con los resultados isotermos antes mencionados.

Como una prueba más de la adecuación de la ecuación (JMA) generalizada para describir experimentos de calenta-

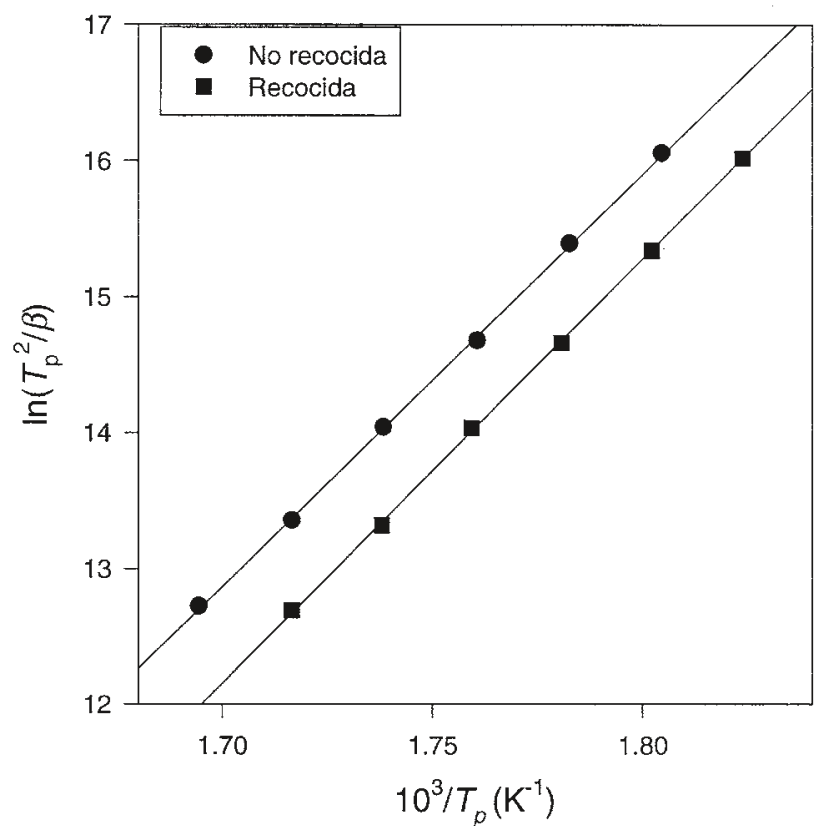

Fig.4. Representación de $\ln \left(T_{\mathrm{p}}{ }^{2} / \beta\right)$ frente $1 / \mathrm{T}_{\mathrm{p}}$ y rectas de regresión para las muestras recocida y no recocida. 


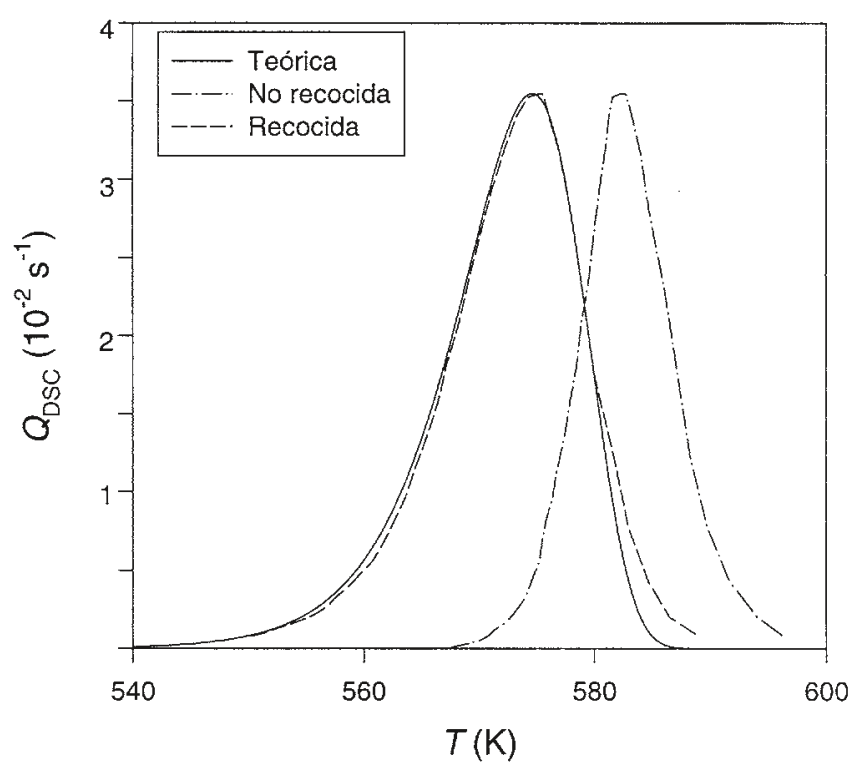

Fig.5. Gráficas de las velocidades de reacción teórica y experimentales para $\beta=32 \mathrm{~K} \mathrm{~min}^{-1}$. Los parámetros cinéticos usados en la curva teórica son los obtenidos por el tratamiento isotermo. El área de la curva teórica se ha normalizado a la unidad. Las alturas de los picos de las curvas experimentales se escalaron para igualar la curva teórica

miento continuo, se han determinado las posiciones y las formas de los picos DSC a partir de la ec.[5], usando los valores de $E, K_{0}, \mathrm{y} n$ obtenidos por los tratamientos isotermos. La Fig.5 muestra la curva teórica típica y las experimentales de procesos no isotermos, incluyendo el efecto del recocido durante un tiempo equivalente al de incubación. Generalmente, las posiciones de los picos DSC de muestras recocidas están en el intervalo de $2 \mathrm{~K}$ de las posiciones teóricas, su anchura total a la mitad del máximo (ATMM) está en $1 \mathrm{~K}$ y se observa la asimetría prevista teóricamente. Las muestras no recocidas presentan picos DSC más estrechos y menos asimétricos que los dados por la teoría JMA generalizada. La Tabla I recoge los valores teóricos y experimentales de $T_{\mathrm{p}}$ y ATMM del material tanto recocido como sin recocer, a las velocidades de calentamiento empleadas en este estudio. Debe señalarse que las curvas teóricas de calentamiento continuo se han basado en el mejor ajuste posible a los parámetros cinéticos isotermos y presentan una asimetría tal, que la semiordenada máxima derecha dista de la máxima $2 / 3$ aproximadamente de lo que dista la semiordenada máxima izquierda. Esta asimetría no se observa en muestras no recocidas [Exp.(a)] pero está presente en las muestras recocidas [Exp.(b)].

\section{CONCLUSIONES}

La teoría JMA, que proporciona una descripción adecuada de las transformaciones isotermas, se ha adoptado como base para el estudio de transformaciones no isotermas, realizando la generalización apropiada para una variación arbitraria de la temperatura y del tiempo. Se ha admitido que la fracción transformada $x(t)$ debe ser una funcional de la función $T\left(t^{\prime}\right)$ para todo $t^{\prime}<t$, como sucede al analizar la evolución de una reacción a lo largo de un camino arbitrario en un diagrama T-T-T (19). Utilizando la ecuación generalizada se ha obtenido una expresión para calcular los parámetros cinéticos $E$ y $K_{0^{\prime}}$ que coincide con la ecuación de Kissinger y está de acuerdo con el análisis de Henderson (9).
El método teórico desarrollado se ha aplicado a los datos experimentales de la aleación vítrea $\mathrm{Cu}_{0.10} \mathrm{As}_{0.40} \mathrm{Se}_{0.50}$, observándose una buena similitud entre los valores de los parámetros cinéticos obtenidos a partir de medidas isotermas y no isotermas, especialmente en aquellas muestras en las que se han eliminado los efectos de incubación mediante recocido. Los errores de $E$ y $\ln K_{0}$ son inferiores al $2.7 \%$, que está dentro del límite de error experimental. También se han usado los parámetros cinéticos calculados a partir de los datos isotermos para predecir curvas DSC no isotermas. El buen acuerdo entre las temperaturas de pico, $T_{\mathrm{p}^{\prime}}$ las anchuras totales a la mitad del máximo (ATMM) y las asimetrías de las curvas experimentales y predicha son una prueba más de la fiabilidad del método teórico desarrollado.

\section{AGRADECIMIENTOS}

Los autores agradecen a la Junta de Andalucía y a la CICYT $\mathrm{su}$ apoyo financiero (Proyecto $\mathrm{n}^{\mathrm{o}}$ MAT98-0791)

\section{BIBLIOGRAFÍA}

1. H.E. Kissinger, "Reaction Kinetics in Differential Thermal Analysis". Analyt. Chem. 29, 1702-1706 (1957).

2. F.E. Luborsky and H.H. Liebermann, "Crystallization Kinetics of Iron-Boron Amorphous Alloys". Appl. Phys. Lett. 33, 233-234 (1978).

3. K.H.J. Buschow, and N.M. Beekmans, "Thermal Stability of Amorphous cobalt-based Alloys". Phys. Status Solidi A 60, 193-200 (1980).

4. M. Matsuura, "Crystallization Kinetics of Amorphous Iron-Boron Alloys by DTA". Solid St. Commun. 30, 231-233 (1979).

5. J. Vázquez, P.L. López-Alemany, P. Villares and R. Jiménez-Garay, “A Study on Glass Transition and Crystallization Kinetics in $\mathrm{Sb}_{0.12} \mathrm{As}_{0.36} \mathrm{Se}_{0.52}$ Glassy Alloy by Using Non-Isothermal Techniques". Mater. Chem. Phys. 57, 162-168 (1998).

6. J. Vázquez, P.L. López-Alemany, P. Villares and R. Jiménez-Garay, “A Study on the Thermal Stability of some alloys in the Cu-Ge-Te Glassy System by Differential Scanning Calorimetry". Thermochim. Acta 327, 191-196 (1999).

7. J. Vázquez, P.L. López-Alemany, E. Márquez, P. Villares and R. Jiménez-Garay, "A Comparative Study of Activation Energies Calculated from NonIsothermal Transformations of Glassy Alloys". J. of Res. in Phys. 27, 167-175 (1998).

8. P.L. López-Alemany, J. Vázquez, P. Villares and R. Jiménez-Garay, “Kinetic Study on Non-Isothermal Crystallization in Glassy Materials: Application to the $\mathrm{Sb}_{0.12} \mathrm{As}_{0.40} \mathrm{Se}_{0.48}$ Alloy". J. of Alloys and Compounds 285, 185-193 (1999).

9. D.W. Henderson, “Thermal Analysis of Non-Isothermal Crystallization Kinetics in Glass Forming Liquids". J. Non-Cryst. Solids 30, 301-315 (1979).

10. L. Granasy and T. Kemeny, “The Nonexistence of Dynamic Correction Term in the Evaluation of Nonisothermal Measurements [on Metallic Glass] ". Thermochim. Acta 42, 289-295 (1980).

11. W.A. Johnson and R.F. Mehl, "Reaction Kinetics in Processes of Nucleation and Growth". Trans. Am. Inst. Min. Engrs 135, 416-442 (1939).

12. M. Avrami, "Kinetics of Phase Change. I. General Theory". J. Chem. Phys. 7, 1103-1112 (1939).

13. M. Avrami, "Kinetics of Phase Change. II. Transformation-Time Relations for Random Distribution of Nuclei". J. Chem. Phys. 8, 212-224 (1940).

14. M. Avrami, "Kinetics of Phase Change. III. Granulation, Phase Change, and Microstructure". J. Chem. Phys. 9, 177-184 (1941).

15. J. Vázquez, C. Wagner, P. Villares and R. Jiménez-Garay, "A Theoretical Method for Determining the Crystallized Fraction and Kinetic Parameters by DSC, Using Non-Isothermal Techniques". Acta Mater. 44, 4807-4813 (1996).

16. H. Yinnon and D.R. Uhlmann, "Applications of Thermoanalytical Techniques to the Study of Crystallization Kinetics in Glass-Forming Liquids". J. NonCryst. Solids 54, 253-275 (1983).

17. C. Wagner, J. Vázquez, M. Domínguez, P. Villares and R. Jiménez-Garay, "Analysis of the Crystallization Kinetics in the Semiconducting glassy alloy $\mathrm{Cu}_{0.20} \mathrm{As}_{0.35} \mathrm{Te}_{0.45}$ ". Mater. Chem. Phys 43, 227-232 (1996).

18. Z. Altounian, Tu Guo-Hua, J.O.Strom-Olsen and W.B. Muir, "Crystallization of Amorphous Copper-Zirconium $\left(\mathrm{CuZr}_{2}\right)^{\prime \prime}$. Phys. Rev. B 24, 505-509 (1981).

19. N. Clavaguera, "Non-equilibrium Crystallization, Critical Cooling Rates and Transformation Diagrams". J. Non-Cryst. Solids 162, 40-50 (1993). 